\title{
A physiotherapy triage service for orthopaedic upper limb service: an effective way to reduce clinic visits and identify patients for operative intervention
}

\author{
Rebecca F. Lyons ${ }^{1}$ (D) Mary Cassells ${ }^{1} \cdot$ Darragh Hynes $^{1} \cdot$ Hannan Mullett ${ }^{1} \cdot$ Patrick J. Groarke $^{1}$
}

Received: 7 September 2020 / Accepted: 20 March 2021 / Published online: 27 May 2021

(c) Royal Academy of Medicine in Ireland 2021

\begin{abstract}
Introduction Waiting times for orthopaedic outpatient clinics are steadily increasing over the past number of years worldwide. Physiotherapy triage clinics are being utilised to improve access for patients with non-urgent or routine musculoskeletal disorders, to be seen in a timely manner in specialised out-patient clinics. Using these clinics, the aim is to ultimately stratify patients into appropriate management pathways. The aim of our study is to review the effectiveness of a physiotherapy triage clinic run by advanced practitioner physiotherapists (APP), who specialise in the review of upper limb referrals from primary care physicians.

Methods For this study, a prospective, observational design was used. Patients were referred to the Department of Orthopaedic Surgery, Upper Limb Service at a national elective Orthopaedic Unit. The patients' referrals were reviewed and allocated to a physiotherapy triage pathway if deemed routine, non-urgent cases. After assessment in the APP clinic, the physiotherapist made recommendations and highlighted patients who required review or case discussion with an orthopaedic surgeon. The discharge rate and outcome of patients referred on for further interventions or operative procedures was followed over a 3-year period. The outcomes for the patients were reviewed, including whether patients who met an orthopaedic surgeon went on to have surgical intervention.

Results During the study, 646 patients were reviewed in an upper limb APP physiotherapy triage clinic. Of those reviewed, only 201 patients required review by an orthopaedic surgeon. Of those, 56 patients were scheduled for an operative procedure. Within the 3-year period, 50 patients of those scheduled underwent the procedure. The most commonly performed procedure being an arthroscopic subacromial decompression with or without acromioplasty or rotator cuff repair. A total of 145 patients referred by the physiotherapist had a shoulder injection including subacromial and glenohumeral intra-articular injection. The initial discharge rate was $68 \%$.

Conclusions There is a high initial discharge rate after initial assessment by APP triage clinics for upper limb musculoskeletal pathology. This is beneficial in alleviating waiting list pressures allowing only those patients in need of intervention to be placed on the ever expanding waiting lists to see orthopaedic surgeons. This study shows a high proportion of patients being offered surgical intervention after being referred by the APP. We conclude from this that the agreement between the physiotherapist's initial diagnosis and that of the consultant surgeon being similar in identifying patients who would benefit from operative intervention.
\end{abstract}

Keywords Advanced Practitioner Physiotherapist · Orthopaedic Surgery · Outpatient clinic · Physiotherapy · Service provision

Rebecca F. Lyons

rebeccalyons1@hotmail.com

1 National, Orthopaedic Hospital, Cappagh, Finglas, Dublin, Ireland

\section{Introduction}

Worldwide, there is a rising prevalence of musculoskeletal disorders; this has impacted on waiting lists globally. Patients with routine, non-urgent disorders can wait months to years for an appointment to see a specialist orthopaedic surgeon. Often, these patients do not require specialist 
surgical or medical intervention. While on the waiting list, they have no improvement of their quality of life or symptom control. In order to reduce the waiting times, physiotherapy-led triage clinics have become common practice worldwide $[1,2]$. The advanced practice physiotherapists (APP) or extended scope physiotherapists (ESP) can review the patient and identify those in need of further imaging, intervention or operative procedures. In many institutions worldwide, the APP administer injections and, in some countries, can list patients for surgical procedures [3].

In 2012, the Republic of Ireland introduced the first cohort of clinical specialist physiotherapists or APP's. This was a joint initiative of the national clinical programme for both orthopaedic surgery and rheumatology. Twenty-four APP posts were developed at that time. The service aimed to triage the care of patients on waiting lists for musculoskeletal care. Referral letters were reviewed by the Orthopaedic or Rheumatology department; patients deemed to warrant a non-urgent or routine appointment were offered an APP musculoskeletal appointment to facilitate faster access to specialised care [2, 3].

Within the Irish hospital setting, the role of the APP is to triage the patient and investigate or diagnose the underlying pathology. Advice and education about the condition are given. The patient will be referred for a program of physiotherapy, which, in many cases, can improve symptoms. If the patient fails this non-operative treatment pathway, the APP can refer onto the appropriate surgeon.

In our unit, most APP's have completed injection therapy training and are competent doing all shoulder injections. Currently, APP's do not order advanced diagnostic imaging studies or list patients for operative procedures. However, they do liaise with the relevant medical team to arrange these. Worldwide, APP's do list patients for operative procedures, especially related to hip and knee pathology [4-6].

One of the primary goals of the APP outpatient clinics is to reduce patient waiting times for orthopaedic and rheumatology appointments; this has been proven in numerous papers globally $[2,4]$. This is one of the most important outcomes, as ultimately it improves patient satisfaction with the service and also can improve patient care and quality of life [6].

This study was devised to look at the effectiveness of an APP-led orthopaedic triage clinic for upper limb pathology. Back pain and arthroplasty APP clinic outcomes are well published $[5,11,13]$. However, within the field of upper limb pathology, the literature is sparse, particularly when looking at the operative rate from initial review at an APP clinic $[5,11,13]$.

Therefore, our main objective included assessing the discharge rates after initial review or injection therapy. The percentage of patients who went on to have an operative procedure based on the physiotherapist's referral was recorded. A review of the number of patients who had injection therapy completed by trained physiotherapists and the need for subsequent follow-up after this was undertaken.

\section{Methods}

Over a 3-year period, patients seen in a specialised upper limb APP orthopaedic clinic were reviewed through a prospectively maintained database on a password-locked computer. Between 2013 and 2016, 646 non-urgent or routine referrals from general practitioners were triaged to attend the specialised physiotherapy-led clinic by the treating upper limb surgeons. Patients were categorised as urgent or routine. This was based on the referral letter and diagnosis.

At APP-led clinics, patients were examined by the specialist physiotherapist. A detailed history and examination were completed. Advanced imaging was organised if necessary.

Patients deemed suitable for injection of either the subacromial space or glenohumeral joint were discussed with the upper limb orthopaedic consultant. This included patients with a diagnosis of subacromial bursitis, impingement, chronic rotator cuff disease or adhesive capsulitis. The trained physiotherapist then completed these injections once approval was gained. Any patient listed for injection therapy was also referred for formal physiotherapy also. If the physiotherapist believed a patient warranted operative intervention, the case was discussed and reviewed in an orthopaedic outpatient clinic with 3 weeks of initial review. The patient would be listed for an operative intervention after this review, by the treating surgeon if necessary. The outcome of patients referred for surgical intervention was followed. This facilitated analysis of the correlation between the physiotherapist and treating surgeon with regards to a recommendation of operative intervention.

Using Microsoft Xcel, a password-protected database of all patients seen in the APP clinic was maintained. All patients referred for consideration of operative intervention were contacted by phone for follow-up of the outcome. All patients referred for physiotherapy were followed up prospectively at 3 and 6 months by phone. A review of symptoms and need for further investigations or interventions was recorded. All patients post-injection were followed up by phone at 6 weeks post-injection. Outcome post-injection was recorded as discharge due to symptom resolution or repeat assessment. Outcomes were recorded on the passwordlocked Microsoft Xcel database.

\section{Results}

Between 2013 and 2016, 646 new patients were assessed in the Musculoskeletal Upper Limb APP clinic. These patients were deemed as routine, non-urgent reviews for upper limb pathologies. Of the 646 patients reviewed, 445 (68.8\%) were 
discharged with a formal physiotherapy referral and education. Formal physiotherapy was obtained within 3 weeks of referral for all patients. Only 15\% of those discharged to physiotherapy represented to the service in the study time frame between 2013 and 2016.

A total of 201 (31.2\%) were referred on to an upper limb orthopaedic surgeon. Of these 201 patients, the outcomes included injection, performed by a physiotherapist or doctor and placing the patient on a waiting list for an operative intervention.

The APP discussed 145 patients' cases with the orthopaedic surgeon; these went on to receive injection therapy followed by a rehabilitation programme consisting of physical therapy and education. Injection therapy and formal physiotherapy were completed within 3 weeks of the decision being made. Of these 145 cases, 79 patients (54.5\%) received a subacromial injection, given by the APP. Sixtysix patients received glenohumeral, subacromial or a combination of injections given by an orthopaedic doctor. Six weeks post-injection, all patients were contacted by phone, to assess symptom improvement or need for further review. The overall discharge rate after the initial injection was 93.1\% ( $n=135)$ (Fig. 1).

Only $7 \%$ of those treated with injection therapy needed further follow-up and repeat injection in the 72-month follow-up period. Of all the patients initially treated with injection therapy, 5 patients (3.4\%) went on to definitive surgical intervention.

Fifty-six patients (27.8\%) of the 201 patients, directly referred to the orthopaedic surgeon by the APP, were listed for operative intervention. The surgical outcomes were followed for period of 72 months from the start of the study. In this timeframe, 50 patients of the 56 listed for surgery were completed. Four patients were cancelled pre-operatively as symptoms had resolved, one patient did not attend and one patient was deferred due to concurrent medical issues.

Of the surgeries completed, the majority had shoulder arthroscopy, subacromial decompression and acromioplasty $(n=29,58 \%)$ for a diagnosis of shoulder impingement. Eighteen percent $(n=9)$ of patients underwent shoulder joint

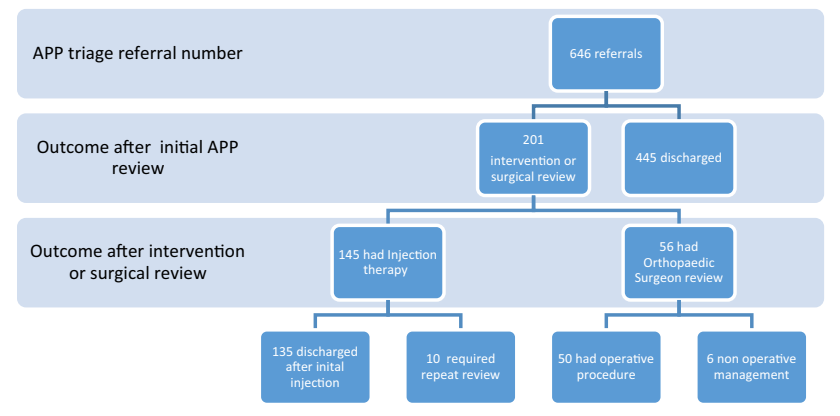

Fig. 1 Flow chart of patient pathway after triage clinic arthroplasty including reverse geometry arthroplasty for a diagnosis of rotator cuff arthropathy. Four arthroscopic stabilisations and one Latarjet were completed for multidirectional glenohumeral instability. Five arthroscopic capsular releases for chronic adhesive capsulitis and 2 rotator cuff repairs were also completed (Fig. 2).

\section{Discussion}

Currently, the burden of musculoskeletal disorders is increasing. This is having a detrimental effect on waiting times to see musculoskeletal specialists such as orthopaedic surgeons and rheumatologists. Prolonged waiting lists lead to poor patient satisfaction and quality of life for those affected. The role of physiotherapists is changing in the modern healthcare system. Their role is now more specialised. These highly trained allied health care professionals can be utilised to aid in decreasing waiting list times [1-5]. In specialised orthopaedic and rheumatology clinics, advanced practitioner physiotherapists (APP) or extended scope physiotherapists (ESP) review and manage patients. These specialised clinics can facilitate the review of large numbers of patients for both new and review appointments. In Ireland, in 1 year, over 30,000 patients were taken off routine and non-urgent orthopaedic and rheumatology waiting lists. Research surrounding APP clinics for hip, knee and back pain is plentiful. The literature reports successful outcomes for both the health service and the patients [2,3]. However, literature surrounding shoulder care pathways is limited. This is concerning since upper limb pathology is a common cause

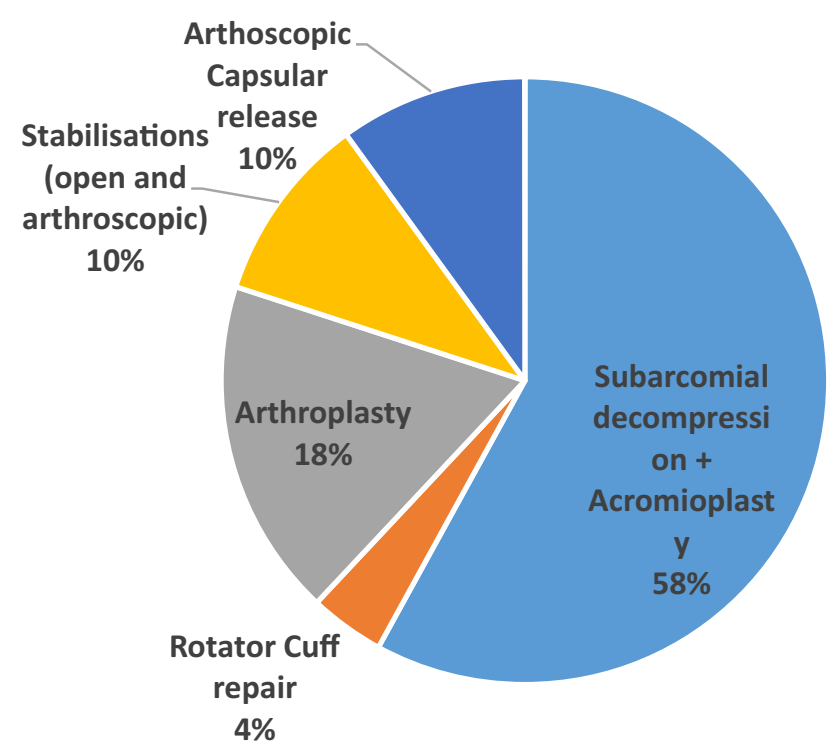

Fig. 2 Pie chart of surgical procedures completed 
of referral to tertiary centre specialist surgeons. In one study, shoulder pathology made up to $15 \%$ of referrals of all new patients presenting for review to orthopaedic clinics $[2,4,5,8]$.

The aims of these clinics are to facilitate timely review of patients and to manage their condition appropriately. As we have shown in our study, these clinics provide a high discharge rate from the first review and formal physiotherapy referral. This is consistent throughout the literature, as most of the patients presenting have not had physiotherapy before referral to the orthopaedic services $[4,7]$. Harrison et al. proved that up to $50 \%$ of patients who were seen in a physiotherapy-led clinic were treated with physiotherapy alone and discharged [8]. By referring these patients onto the appropriate pathway of physiotherapy at their initial assessment, we are creating more clinic appointments for patients who need to see an orthopaedic surgeon specifically. This has a knock-on effect of decreasing the waiting time. This is of particular importance now as we enter a new era of medicine due to the impact of Covid 19. Covid 19 has highlighted the need for improved patient pathways in the healthcare setting. Worldwide, we are striving to make healthcare services more efficient by reducing the number of interactions between the patient and healthcare provider. This study shows that this APP pathway can effectively triage and treat patients, reducing the amount of times they must attend the hospital.

Physiotherapists are highly skilled allied health care professionals. In order to utilise all the skills, these professionals have to offer, training in areas such as injection therapy can have major benefits in reducing time to treatment in upper limb conditions. We have shown that $54 \%$ of patients who required injection therapy, received it from the physiotherapist within 3 weeks of initial review. Previously, these patients would have been booked into a second orthopaedic clinic to be assessed by the surgeon and subsequently injected by a doctor. Training the APP to inject the subacromial space and glenohumeral joint is a valuable asset to an orthopaedic unit and decrease the time to injection for a patient.

Previously, there were concerns about physiotherapists listing patients for operative procedures and doing injections. However, literature is currently showing positive correlation between the decisions experienced physiotherapists and surgeons make about patient care and management options. Marks et al. showed that physiotherapists and orthopaedic surgeons reviewing patients with shoulder pain made nearly identical decisions about surgical and non-surgical options for patients and the need for advanced imaging. It also showed that physiotherapists were moderately less inclined to give an injection to a patient at initial review compared with the surgeon [7]. This highlights that with the appropriate training, physiotherapists can safely identify and inject patients with shoulder pain, who have not been seen by an orthopaedic surgeon.
The literature surrounding APP orthopaedic clinics investigate different elements of the service provision. This includes decision making as described in our paper. The majority of the literature discusses clinical decision making with reference to hip and knee pathology. However, from published literature, there is strong correlation between surgeons and physiotherapists with regards to clinical diagnosis and management decisions [9-11]. Fewer publications look at the correlation between practitioners for upper limb pathology. Razmjou et al. reported that the agreement between an upper limb surgeon and physiotherapist had a kappa score of $0.63-0.68$ for diagnosis and 0.75 for considering surgery as a treatment option [12]. This correlation is lower than for hip and knee conditions. This highlights the need for further education of experienced physiotherapists who would be running these APP upper limb triage clinics. By increasing the education and engagement of upper limb consultants in this process, there is no doubt that the outcomes seen in hip and knee clinics could be replicated for upper limb pathology also.

One of the primary aims of this paper was to look at the discharge rate after initial reviews and if those patients represented again. We have shown a large proportion of our upper limb patients were indeed discharged after their initial review with physiotherapy referral. Only $7 \%$ needed further review after formal physiotherapy. Those patients who were discharged included patients with a diagnosis of subacromial bursitis, adhesive capsulitis, impingement and chronic rotator cuff tears.

In this study, 56 patients were highlighted as surgical candidates by the APP and sent for review to the upper limb orthopaedic consultant. Of those sent for review, 50 patients underwent an operative procedure in the study timeframe. This is a very successful conversion rate from those referred. Again, this highlights that with further education and training APP may in the future be tasked with listing people directly for surgery for upper limb surgical intervention. Previously, there were concerns about the APP ability to make these kinds of clinical decisions but a number of paper of papers have supported the APP decision making in correlation with their medical colleagues. Further research would be beneficial to assess this correlation for upper limb pathology. Currently, however, the literature does support the ability of the APP to demonstrate the clinical judgement needed $[14,15]$.

One of the limitations of this study is that we did not formally assess the patient satisfaction using any standardised patient-related outcome measures. However patient satisfaction has been proven in the literature to be high after this style of intervention. Patients with hip and knee pathology treated with physiotherapy exercises and advice report improvement in all self-efficacy scales 6 weeks after their first APP clinic visit $[13,16]$. It is clear that further research into patient 
satisfaction with upper limb pathology would be beneficial to prove the effectiveness in this cohort of patients also.

This study highlights that triage via the APP/ESP pathway is a safe and effective treatment course for conditions of the upper limb. When referring a patient on to an orthopaedic outpatient for consideration for surgery, there was a high level of agreement. This pathway allows more timely access to a competent healthcare professional and effectively treats the majority of patients without requiring a surgical opinion. This study shows the success of this treatment pathway. It can serve to encourage general practitioners and patients that it can reduce symptoms effectively while reducing waiting times for such treatments.

Author contribution RF Lyons: Data analysis and document preparation. M Cassells: Data collection and patient interaction. D Hynes: Proof reading and document preparation. H Mullett: Concept design and document preparation. PJ Groarke: Concept design and document preparation.

\section{Declarations}

Ethical approval The authors would like to confirm local ethical approval and consent was obtained for completion of the research. We, the authors, have no competing interests to declare and no additional funding was obtained.

\section{References}

1. Morris J, Twizeyemariya A, Pillen H, Grimmer K (2017) What happens when patients with an orthopaedic complaint 'wait'? Setting the scene in one Australian tertiary. Asia Pac J Health Manag 12:34-41

2. Fennelly O, Blake C, FitzGerald O et al (2018) Advanced practice physiotherapy-led triage in Irish orthopaedic and rheumatology services: national data audit. BMC Musculoskelet Disord 19:181. https://doi.org/10.1186/s12891-018-2106-7

3. Fennelly O, Blake C, Desmeules F et al (2017) Patient reported outcome measures in advanced musculoskeletal physiotherapy practice: a systematic review. Musculoskelet Care 16:188-208
4. Pearse EO, Maclean A, Ricketts DM (2006) The extended scope physiotherapist in orthopaedic out-patients - an audit. Ann R Coll Surg Engl 88(7):653-655

5. Wood L, Hendrick P, Boszczyk B, Dunstan E (2016) A review of the surgical conversion rate and independent management of spinal extended scope practitioners in a secondary care setting. Ann R Coll Surg Engl 98(3):187-191

6. Stanhope J, Grimmer-Somers K, Milanese S et al (2012) Extended scope physiotherapy roles for orthopedic outpatients: an update systematic review of the literature. J Multidiscip Healthc 5:37-45. https://doi.org/10.2147/JMDH.S28891

7. Marks D, Comans T, Thomas M et al (2016) Agreement between a physiotherapist and an orthopaedic surgeon regarding management and prescription of corticosteroid injection for patients with shoulder pain. Man Ther 26:216-222

8. Harrison J, Rangan A, Shetty A, Robinson C (2001) Reducing waiting times: physiotherapy shoulder assessment clinic. Brit J Ther Rehabil 8:57-59

9. Parsons S, Breen A, Foster NE et al (2007) Prevalence and comparative troublesomeness by age of musculoskeletal pain in different body locations. Fam Pract 24(4):308-316

10. Aiken AB, McColl MA (2008) Diagnostic and treatment concordance between a physiotherapist and an orthopedic surgeon - a pilot study. J Interprof Care 22:253-261

11. Desmeules F, Toliopoulos P, Roy JS et al (2013) Validation of an advanced practice physiotherapy model of care in an orthopaedic outpatient clinic. BMC Musculoskelet Disord 14:162

12. Oldmeadow LB, Bedi HS, Burch HT et al (2007) Experienced physiotherapists as gatekeepers to hospital orthopaedic outpatient care. Med J Aust 186:625-628

13. Razmjou H, Robarts S, Kennedy D et al (2013) Evaluation of an advanced- practice physical therapist in a specialty shoulder clinic: diagnostic agreement and effect on wait times. Physiother Can 65:46-55

14. MacKay C, Davis AM, Mahomed NN, Badley EM (2012) A single group follow-up study of non-surgical patients seen by physiotherapists working in expanded roles in orthopaedic departments: recall of recommendations, change in exercise and self-efficacy. BMC Res Notes 5:669

15. Griffiths S, Taylor C, Yohannes AM (2012) Conversion rates and perceived barriers to referral: views of extended scope physiotherapists in the primary care setting. Musculoskeletal Care 10:221-231

16. Reeve S, May S (2009) Exploration of patients' perspectives of quality within an extended scope physiotherapists' spinal screening service. Physiother Theory Pract 25:533-543 